\title{
Guest editorial to the special issue on UML 2004
}

\author{
Thomas Baar · Ana Moreira
}

Received: 3 March 2006 / Accepted: 3 March 2006 / Published online: 12 July 2006

(C) Springer-Verlag 2006

The UML 2004 was held in Lisbon (Portugal), 11-15, October 2004 and was the seventh conference in the series of annual UML conferences. The 2004 edition of the UML conference proved to be a milestone event since it was the last conference under the UML banner, and the announcement of the new format, MoDELS (MOdel Driven Engineering, Languages and Systems). The decision to continue the highly successful UML conference series under its new name MoDELS was more than a mere name change. The name MoDELS represents a formal acknowledgement of how the conference's scope has broadened over the years. While in the first years the focus of the conference was on the scientific investigation of the Unified Modeling Language (UML), which was adopted by the Object Management Group (OMG) at that time, the focus changed over time to include also new techniques such as metamodeling, model transformations, model validation and verification, model driven architecture and development, aspect orientation and beyond.

The 2004 UML conference attracted 240 participants who had to choose from an extensive variety of events: 30 paper presentations in 10 technical sessions, 3 keynote talks, 2 panel discussions, 10 workshops, including the first Doctoral Symposium, 5 tutorials and, held for the first time, a special track for industry papers

\section{T. Baar $(\bowtie)$}

EPFL/IC/UP-LGL, Station 14, 1015,

Lausanne, Switzerland

e-mail: thomas.baar@epfl.ch

\section{A. Moreira}

CITI/Computer Science Department,

Universidade Nova da Lisboa, 2829-516,

Caparica, Portugal

e-mail: amm@di.fct.unl.pt chaired by Bran Selic. The keynote talks were given by Krzysztof Czarnecki, who offered his insights on "Generative Software Development", by Desmond D'Souza, talking on "Goals, Viewpoints and Components - an MDA Perspective", and by Oscar Nierstrasz, who suggested to improve software quality by "Putting Change at the Center of the Software Process". Besides the items from the conference programme, there were other highlights, such as a Best Paper Award and a post-conference book, that made the UML 2004 conference even more attractive. The Best Paper Award sponsored by Springer recognizes one conference paper for its outstanding scientific contribution. The post-conference book $U M L$ 2004 Satellite Activities, volume 3,297 of Springer's LNCS series, was published in addition to the conference proceedings and contains industry papers, reports on the workshops, and tool/poster presentations.

Based on the quality of the papers and their presentations at the conference, the program committee invited the authors of six papers (including one paper from the industry track) to submit an extended and enhanced version to this special issue. The six submissions went through a rigorous review process. After a first iteration of reviews, the authors were asked to resubmit an improved version addressing issues identified by the reviewers. The second iteration of reviews uncovered in many cases some additional, minor points that have been later resolved by the authors when preparing the final version. For the laborious task of assessing the submissions and giving in many cases, extensive hints for improvements, we sincerely thank all reviewers of this special issue: Fernando Brito e Abreu, Omar Aldawud, João Araújo, Doo-Hwan Bae, Jean Bézivin, Jean-Michel Bruel, Siobhán Clarke, Steve Cook, Gregor 
Engels, Andy Evans, Stephan Flake, Jeff Gray, Heinrich Hussmann, Jean-Marc Jézéquel, Stuart Kent, Tim Lethbridge, Pierre-Alain Muller, Ivan Porres, Wolfgang Reisig, Peter H. Schmitt, Andy Schürr, Ambrosio Toval and Jon Whittle.

Based on the recommendation of the reviewers, the following five papers were selected for publication.

Alexandre Correa and Cláudia Werner: Refactoring OCL Specifications (a shorter version of this paper was the winner of the Best Paper Award at UML 2004). This paper discusses how refactoring techniques can be applied to simplify the structure of OCL specifications and, thus, to improve understandability and maintainability. The authors present several potentially bad structures often found in OCL specifications and a collection of refactorings to overcome them. Finally, the paper discusses how refactorings can be automated and how model regression testing is used to increase the modeler's confidence that the original and the refactored OCL specifications are semantically equivalent. The approach has been implemented by means of a prototypical tool.

Ragnhild Van Der Straeten, Viviane Jonckers and Tom Mens: A Formal Approach to Model Refactoring and Model Refinement. This paper formally defines and explores the relation between behaviour inheritance consistency of a refined model and behaviour preservation of a refactored model, both with respect to the original model. The authors find a close correlation between the notions of behaviour consistency and behaviour preservation and formally demonstrate this similarity. Validation is performed by means of a prototype plug-in in a UML CASE tool.

Simone Röttger and Steffen Zschaler: Tool Support for Refinement of Non-functional Specifications. This paper extends and refines the authors' previous work on model-driven development for non-functional properties. The authors describe measurement refinement and the related models from three perspectives: application designer's view, measurement designer's view and technical, tool-oriented view. The paper also presents a prototype implementation of a tool.
Dorin B. Petriu and Murray Woodside: An Intermediate Metamodel with Scenarios and Resources for Generating Performance Models from UML Designs. This paper defines the Core Scenario Model (CSM) and describes how it resolves questions that arise in performance analysis of software designs. The CSM provides a metamodel for an intermediate form which correlates multiple UML diagrams, extracts the behaviour elements with the performance annotations, and attaches important resource information that is obtained from the UML. The CSM provides, thus, a bridge between UML specifications with the UML Profile for Schedulability, Performance and Time (SPT), and existing techniques to generate performance models.

Michael von der Beeck: Development of Logical and Technical Architectures for Automotive Systems. This is an experience paper presenting a modeling approach developed within a safety-relevant project at BMW Group. The approach supports the development of two related architecture models: a logical architecture which provides a graphical and abstract representation; and a technical architecture which provides both software and hardware representations. The author discusses both positive and negative experiences with the application of the approach.

We would like to thank all authors for submitting their work and for improving it according to the reviewers' comments. A special word of thanks goes to the Editors-in-Chief Bernhard Rumpe and Robert France for providing in SoSyM a vehicle that brings this work to a wider specialist audience.

Thomas Baar (thomas.baar@epfl.ch)

Ana Moreira (amm@di.fct.unl.pt)

Guest Editors 